\title{
A multiwavelength investigation of G24.78+0.08 A2 using observations from VLA ${ }^{\star}$ and VLT-VISIR ${ }^{\star}$
}

\author{
S. Vig ${ }^{1}$, R. Cesaroni ${ }^{1}$, L. Testi ${ }^{1,2}$, M. T. Beltrán ${ }^{3}$, and C. Codella ${ }^{4}$ \\ 1 INAF - Osservatorio Astrofisico de Arcetri, Largo E. Fermi 5, 50125 Firenze, Italy \\ e-mail: sarita@arcetri.astro.it \\ 2 ESO, Karl Schwarzschild str. 2, 85748 Garching, Germany \\ 3 Departament d'Astronomia i Meteorologia, Universitat de Barcelona, Martí i Franquès 1, 08028 Barcelona, Spain \\ 4 INAF - Istituto di Radioastronomia, Sezione di Firenze, Largo E. Fermi 5, 50125 Firenze, Italy
}

Received 18 January 2008 / Accepted 13 June 2008

\begin{abstract}
Context. G24.78+0.08 is a massive star-forming region where three large massive disk-like rotating structures (toroids), namely A1, A2 and C, have been found around massive (proto)stars. In particular, while G24 A1 has been extensively investigated through multiwavelength surveys, G24 A2 has been hitherto less studied.

Aims. We carry out a detailed investigation of G24 A2 and speculate the scenario of the environment in the vicinity of the central exciting object(s).

Methods. We have carried out mid-infrared imaging observations of the G24.78+0.08 region at 11.9 and $18.7 \mu \mathrm{m}$ using the VISIR located on the VLT. In addition, the radio continuum VLA observations at $1.3 \mathrm{~cm}$ as well as the $\mathrm{NH}_{3}(2,2)$ line emission have been investigated.

Results. Compact mid-infrared emission is observed only from one source in the G24.78+0.08 region and this is likely to be associated with G24 A2. The radio continuum measurements show a faint compact source which is resolved out at higher angular resolutions (60 mas); the diameter $D$ of the radio emitting region is estimated to be $1000 \mathrm{AU}<D<2000 \mathrm{AU}$. The spectral index ( 1) points towards the possibility of this emission being from an ionised jet or an $\mathrm{H}$ II region with a density gradient. $\mathrm{The}^{\mathrm{NH}} 3(2,2)$ emission from the main component is optically thick and appears self-absorbed at blue-shifted velocities. The velocity distributions of the $\mathrm{NH}_{3}$ lines can be explained by a motion which is a combination of rotation and expansion. Based on these observations, we speculate that the expansion is due to the outflow (seen in $\mathrm{CO}$ ) with $\mathrm{A} 2$ being the likely origin of this outflow. The comparison of various properties of G24 A1 and G24 A2 like rotation, mass of cores, infrared emission, excitation temperature, suggests that G24 A2 and G24 A1 possibly harbour similar young stellar objects, but the toroid associated with G24 A2 is more inclined than that of G24 A1.
\end{abstract}

Key words. stars: formation - stars: circumstellar matter - ISM: individual objects: G24.78+0.08 A2 - infrared: ISM radio lines: ISM

\section{Introduction}

One of the debates in the astrophysics of star-formation is whether high mass OB stars form in the same way as their lower mass counterparts. The answer to this question is non trivial: as soon as an early type star reaches the zero age main sequence, its powerful radiation pressure should prevent further accretion and thus quit the growth of the stellar mass beyond $\sim 8 M_{\odot}$ (Palla \& Stahler 1993). A variety of theories have been proposed to solve this problem (see Bonnell et al. 2007; Beuther et al. 2007). One of the approaches involves formation of massive stars via non-spherical accretion of matter through disks. To date, there are very few examples of direct detections of disk-like structures from massive $\left(L>10^{3} L_{\odot}\right)$ protostars. However, in recent years growing evidence of the existence of circumstellar disks in B-type (proto)stars has been presented in the literature

\footnotetext{
* Based on observations obtained at the Very Large Array (VLA) of the National Radio Astronomy Observatory (NRAO). NRAO is a facility of the national Science Foundation operated under cooperative agreement by Associated Universities, Inc.

$\star \star$ Based on observations carried out with the Very Large Telescope (VLT) operated by European Southern Observatory (program ID 075.C0611(A)).
}

(see Cesaroni et al. 2007, for a review). This favours formation of massive stars through disk accretion. One of the wellstudied examples is that of G24.78+0.08 where large massive disk-like structures (called toroids) have been found around massive (proto)stars.

G24.78+0.08 (hereafter G24) is a high mass star-forming region located at a distance of $7.7 \mathrm{kpc}$. It comprises a group of young high-mass (proto)stars in different evolutionary phases embedded in their parental cores, as shown by Furuya et al. (2002). These authors detected bipolar ${ }^{12} \mathrm{CO}$ outflows from two of these cores, G24 A and G24 C. Larger scale properties of the molecular cloud associated with G24 have been investigated in detail by Cesaroni et al. (2003) in various molecules like CO, $\mathrm{CS}, \mathrm{CH}_{3} \mathrm{CN}$ as well as in the continuum. The $\mathrm{G} 24$ region was studied for $\mathrm{NH}_{3}(2,2)$ and $(3,3)$ inversion transitions as well as $1.3 \mathrm{~cm}$ continuum by Codella et al. (1997) using the VLA with an angular resolution of $\sim 1^{\prime \prime}$. Follow-up line and continuum observations at high angular resolution $\left(\$ 1^{\prime \prime}\right)$ by Beltrán et al. (2004) have resolved the structure of G24 A into two distinct subcores, A1 and A2 separated by $\sim 1$ '. 5 . Further, based on $\mathrm{CH}_{3} \mathrm{CN}$ studies, they have shown that both these subcores have a velocity gradient perpendicular to the direction of the known ${ }^{12} \mathrm{CO}$ outflow suggesting that they are massive toroids rotating 
about the outflow axis. The methanol and water masers in this region have been explored by Moscadelli et al. (2007). Based on the positions and the line-of sight velocities of these masers towards A1 and A2, they conclude that a similar young stellar object is responsible for the excitation of the masers in both A1 and A2. Of these two subcores, G24 A1 has been studied in detail by Beltrán et al. (2006) establishing for the first time, the simultaneous presence of outflow, rotation, and infall in A1. It has also been found that $\mathrm{A} 1$ hosts a hypercompact $\mathrm{H}$ II region having a shell-structure which is expanding on a very short time-scale of 70 yrs (Beltrán et al. 2007). On the other hand, relatively little is known about its neighbour, G24 A2. In this paper, we explore the nature of $\mathrm{G} 24 \mathrm{~A} 2$, another toroid candidate, using multiwavelength observations from VLA and VLT-VISIR.

We have carried out a detailed investigation of the core associated with A2 using the infrared, millimetre and radio data. In Sect. 2, we summarize the observations and the results are presented in Sect. 3. A discussion on the nature of G24 A2 is presented in Sect. 4 and a comparison of the two neighbours, G24 A1 and A2, is given in Sect. 5. Finally, a short summary is given in Sect. 6.

\section{Observations and available data}

\subsection{VLT-VISIR}

We have carried out mid-infrared observations of G24 using VISIR. VISIR is the ESO-VLT imager and spectrograph for the mid-infrared (Lagage et al. 2004), mounted on the Cassegrain focus of the VLT Unit Telescope 3 (Melipal). The imaging through the PAH2_2 $(11.88 \mu \mathrm{m})$ and Q2 $(18.72 \mu \mathrm{m})$ filters was carried out on the night of 13 July 2005. The airmass at the time of observations was 1.1-1.3, and the optical seeing was better than $1^{\prime \prime}$. Chopping as well as nodding (both in the North-South direction for the G24 region) were carried out in order to minimise the background. The imaging was carried out with a scale of $0 . ' 075$ per pixel leading to a field-of-view of $19 . ' 2 \times 19.2$. The total integration time on the source in each filter was $30 \mathrm{~min}$. The standard stars HD111915 and HD152880 were observed and used for photometric calibration in both bands. The VISIR data reduction pipeline provided by ESO was used to reduce the data.

\subsection{Spitzer data}

The Spitzer Space Telescope ${ }^{1}$ (Werner et al. 2004) was launched in space in August 2003 and consists of a 0.85-m telescope with three cryogenically cooled instruments: InfraRed Array Camera (IRAC), Multiband Imaging Photometer for Spitzer (MIPS) and InfraRed Spectrograph. We have used data from the Legacy projects, GLIMPSE (PI: Churchwell) and MIPSGAL (PI: Carey) in this paper. The GLIMPSE and MIPSGAL images have been obtained using the software "Leopard".

GLIMPSE surveyed part of the Galactic plane in 3.6, 4.5, 5.8 , and $8.0 \mu \mathrm{m}$ with a pixel size of $1^{\prime \prime} .2 \times 1$ '. 2 (Fazio et al. 2004). The resolutions achieved by IRAC are 2'. 4, 2'. 4,2 .' 8 and 3 ". 0 in the 3.6, 4.5, 5.8 and $8.0 \mu \mathrm{m}$ bands, respectively. The fluxes of the mid-infrared emission in G24 A have been extracted from the GLIMPSE More Complete Archive.

The Multiband Imaging Photometer for Spitzer Galactic Plane survey (MIPSGAL) surveyed the sky in identical regions

\footnotetext{
1 This work is based in part on observations made with the Spitzer Space Telescope, which is operated by the Jet Propulsion Laboratory, under NASA contract 1407.
}

as covered by GLIMPSE at 24 and $70 \mu \mathrm{m}$ using the MIPS instrument (Carey et al. 2005). The instrument achieves diffractionlimited resolution of $6^{\prime \prime}$ and $18^{\prime \prime}$ at 24 and $70 \mu \mathrm{m}$, respectively.

\subsection{VLA}

The observations were made on 21 April 2005 with 26 antennas of the NRAO Very Large Array (VLA) in the B-configuration. We measure the $(2,2)$ inversion transition of ammonia and the $1.3 \mathrm{~cm}$ continuum emission. The phase center was set at $\alpha(\mathrm{J} 2000)=18^{\mathrm{h}} 36^{\mathrm{m}} 12^{\mathrm{s}} .66, \delta(\mathrm{J} 2000)=-07^{\circ} 12^{\prime} 10^{\prime}{ }^{\prime} 15$. The $\mathrm{NH}_{3}(2,2)$ line and the continuum have been observed simultaneously by using the correlator in the 2 IF mode with a $3.13 \mathrm{MHz}$ bandwidth centered at the line frequency $(23722.63 \mathrm{MHz})$ attaining a spectral resultion of $0.30 \mathrm{~km} \mathrm{~s}^{-1}$, and a $25 \mathrm{MHz}$ bandwidth centered at $23737.81 \mathrm{MHz}$ to obtain a continuum measurement. Bandpass and phase were calibrated by observing $1832-105$, the flux density scales were derived by observing 3C 286, while pointing was checked on 1743-0.36. All the data editing and calibration were carried out using the tasks in the NRAO AIPS package. Line cubes were produced using natural weighting, and restored with a clean beam of $0 .{ }^{\prime} 4$ or $0 .{ }^{\prime} 8$ (to improve the signal-to-noise ratio) obtaining a final rms noise of $0.4 \mathrm{mJy} / \mathrm{beam}$. The continuum image was obtained by setting ROBUST $=0$ in task IMAGR of AIPS thus attaining a synthesized beam of $0.30 \times 0 . ' 24$ with PA $=-8.7^{\circ}$ and an $\mathrm{rms}$ of $0.15 \mathrm{mJy} / \mathrm{beam}$.

\section{Results}

In this section, we analyse the emission at the mid-infrared and radio wavelengths towards $\mathrm{G} 24 \mathrm{~A} 2$.

\subsection{Mid-infrared emission}

The VISIR images of G24 A at 11.9 and $18.7 \mu \mathrm{m}$ show emission from a single source. The source is unresolved at both wavelength bands (since the $F W H M$ of the source is the same as that observed for the standard star): $F W H M \sim 0$.' $^{\prime}$ at $11.9 \mu \mathrm{m}$ and $\sim 0 . ' 5$ at $18.7 \mu \mathrm{m}$. The emission from G24 A in these bands, smoothed to a pixel size of $0 . ' 15$ is shown in Fig. 1. The fluxes have been extracted using aperture photometry with an aperture radius of $1^{\prime \prime}$ and the background annulus is of thickness $1^{\prime \prime}$ located at a distance of $4^{\prime \prime}$ from the centre of the aperture. The calibration of fluxes has been carried out using the standard stars. These VISIR mid-infrared fluxes are listed in Table 1. The error in photometry is $\sim 15 \%$, mainly due to uncertainity in the fluxes of the standard stars as well as due to airmass corrections. Although the angular resolution of the VISIR images is high, the astrometry of these images relies on the Spitzer images. As already mentioned in Sect. 1, G24 A comprises two subcores, A1 and A2 separated by $\sim 1 . " 5$. The mid-infrared emission from Spitzer-GLIMPSE suggests that the emission is most likely from A2 as the astrometric uncertainity of the GLIMPSE image is 0 .' $^{\prime}$. Considering the proximity of the mid-infrared emission towards A2, in the following we assume this emission to be associated with A2.

An investigation into the Spitzer-GLIMPSE images shows the presence of mid-infrared emission in all the four IRAC bands at 3.6, 4.5, 5.8 and $8.0 \mu \mathrm{m}$. Figure 2 shows the IRAC 3.6 and $8 \mu \mathrm{m}$ emission as grayscale with the locations of A1 and A2 shown as $1.4 \mathrm{~mm}$ continuum contours as observed by 


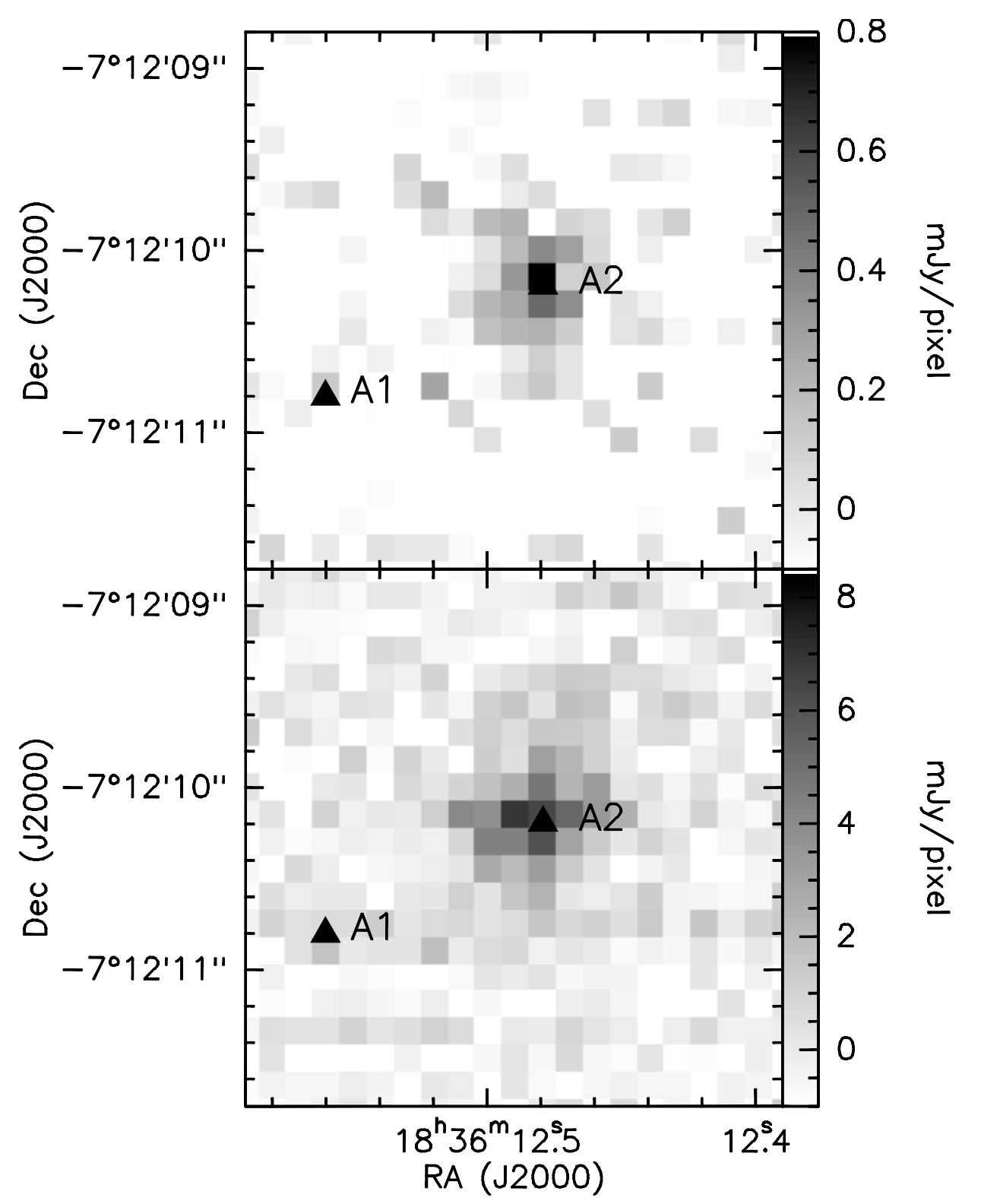

Fig. 1. Image of G24 A at (top) $11.9 \mu \mathrm{m}$ and (bottom) $18.7 \mu \mathrm{m}$ taken with VLT-VISIR.

Table 1. Mid-infrared fluxes of G24.78-0.08 A2 for no dereddening applied $\left(A_{\mathrm{V}}=0 \mathrm{mag}\right)$ and for a dereddening of $A_{\mathrm{V}}=14 \mathrm{mag}$.

\begin{tabular}{ccccc}
\hline \hline Wavelength & Instrument & Aperture & \multicolumn{2}{c}{ Flux (Jy) } \\
$(\mu \mathrm{m})$ & & radius (") & $A_{\mathrm{V}}=0$ & $A_{\mathrm{V}}=14$ \\
\hline 3.6 & Spitzer-IRAC & 2.4 & 0.007 & 0.015 \\
4.5 & Spitzer-IRAC & 2.4 & 0.14 & 0.28 \\
5.8 & Spitzer-IRAC & 2.4 & 0.35 & 0.67 \\
8.0 & Spitzer-IRAC & 2.4 & 0.19 & 0.35 \\
11.9 & VLT-VISIR & 1.0 & 0.08 & 0.13 \\
18.7 & VLT-VISIR & 1.0 & 0.11 & 0.12 \\
\hline
\end{tabular}

Beltrán et al. (2004). Spitzer-GLIMPSE fluxes along with the apertures are listed in Table 1.

The MIPSGal images at $24 \mu \mathrm{m}$ and $70 \mu \mathrm{m}$ (not shown here) are saturated towards G24 A. The flux of the source associated with G24 A is determined to be $>4$ Jy. This flux represents a lower limit as it includes the pixels affected by saturation. Moreover, it is difficult to disentangle the contributions of
G24 A1 and G24 A2. Therefore the 24 and $70 \mu \mathrm{m}$ fluxes will not be used in our analysis.

\subsection{Continuum emission}

The radio continuum emission at $1.3 \mathrm{~cm}$ towards $\mathrm{G} 24$ region is shown as grayscale in Fig. 3. The figure shows the locations of G24 A1, A2, B and C. In particular, G24 A2 is shown as contours in Fig. 4 (top). For comparison, the figure also shows $1.4 \mathrm{~mm}$ continuum measurements made towards A2 by Beltrán et al. (2005). We note that this radio continuum map is an improvement (in terms of angular resolution) over the previous $1.3 \mathrm{~cm}$ measurements. The unresolved free-free emission peaks at $\alpha(\mathrm{J} 2000)=18^{\mathrm{h}} 36^{\mathrm{m}} 12^{\mathrm{s}} 479, \delta(\mathrm{J} 2000)=-07^{\circ} 12^{\prime} 10^{\prime} \cdot 19$. The radio flux at $1.3 \mathrm{~cm}$ is $0.86 \pm 0.07 \mathrm{mJy}$. Moscadelli et al. (2007) studied $44 \mathrm{GHz}$ Class I methanol masers towards the $\mathrm{G} 24$ region. These authors also carried out radio continuum interferometric measurements (using the VLA) at $7 \mathrm{~mm}$ (not shown in their paper). An investigation of this $7 \mathrm{~mm}$ continuum image shows a 


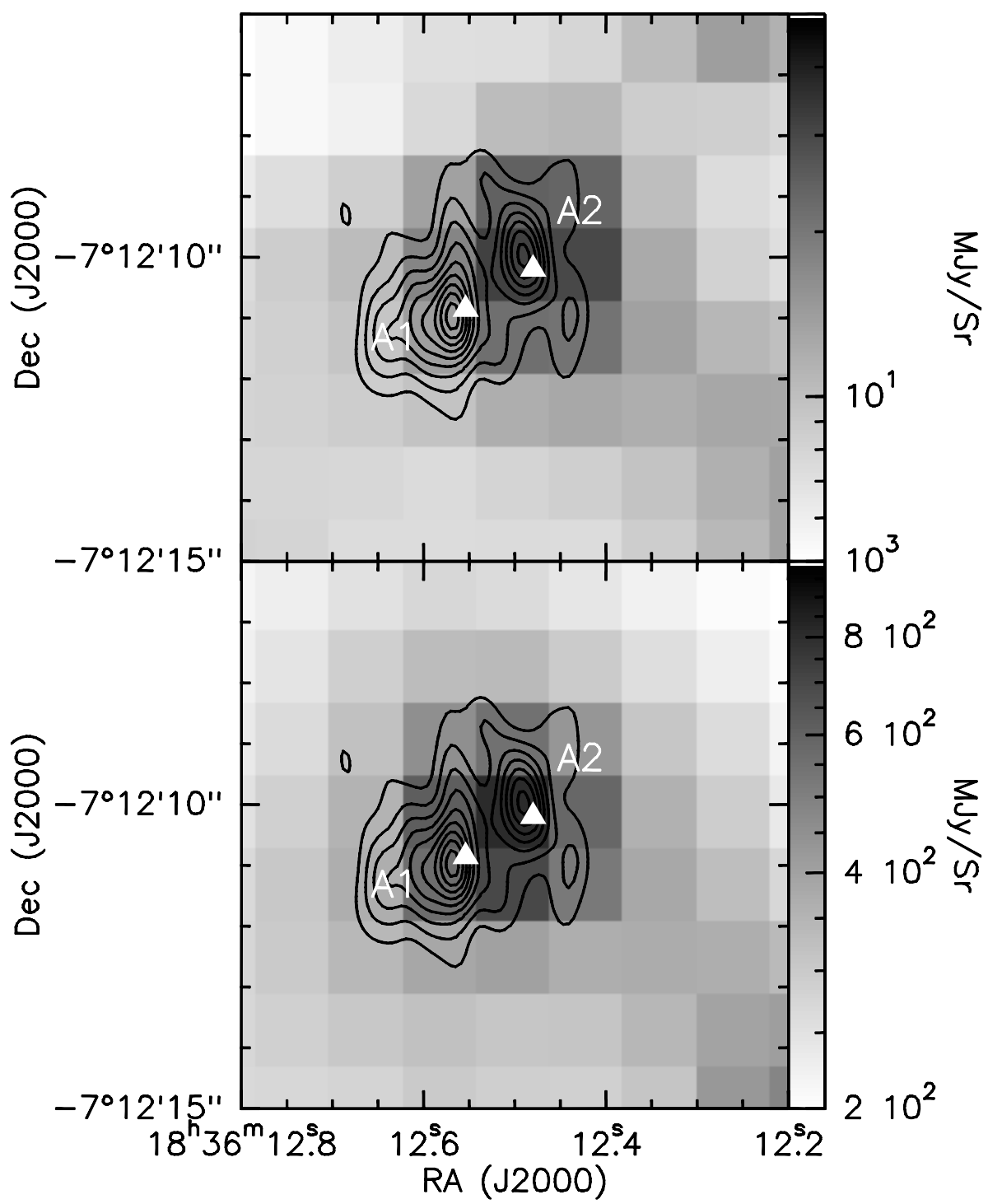

Fig. 2. The image is the IRAC $3.6 \mu \mathrm{m}$ (top) and IRAC $8 \mu \mathrm{m}$ (bottom) of the region around G24 A. The contours are the $1.4 \mathrm{~mm}$ contours from Beltrán et al. (2004) with the symbols marking A1 and A2 (identified by the peak of radio continuum emission at $1.3 \mathrm{~cm}$ ). Contour levels range from 0.04 to $0.2 \mathrm{Jy} /$ beam in steps of $0.02 \mathrm{Jy} / \mathrm{beam}$, where the beam is $1.2 \times 0.2$.
5- $\sigma$ detection at a location that coincides with the radio continuum emission at $1.3 \mathrm{~cm}$. The flux at $7 \mathrm{~mm}$ is $3.0 \pm 0.5 \mathrm{mJy}$.

\section{3. $\mathrm{NH}_{3}(2,2)$ line emission}

The interferometric observations of the $\mathrm{NH}_{3}(2,2)$ inversion transition with 0 .' $^{\prime} 4$ angular resolution allow us to probe the molecular gas around the subcore A2. Earlier interferometric observations of $\mathrm{NH}_{3}(2,2)$ with the VLA by Codella et al. (1997) attained only an angular resolution of $\sim 1^{\prime \prime}$ and resolved G24 A and G24 B, but not the subcores, A1 and A2. The $\mathrm{NH}_{3}(2,2)$ inversion transitions consist of a number of hyperfine components distributed in such a way to form a "main" line symmetrically surrounded by four "satellites". The $\mathrm{NH}_{3}$ emission towards the full $\mathrm{G} 24$ region (including $\mathrm{A} 1, \mathrm{~A} 2, \mathrm{~B}$ and $\mathrm{C}$ ) is shown as contours in Fig. 3 while the emission towards G24 A2 is shown as grayscale in Fig. 4. The $\mathrm{NH}_{3}(2,2)$ spectrum averaged over the entire A2 emission region is shown in Fig. 5. The correlatorsetup of the VLA allows us to probe the main line as well as the two inner satellites. These can be seen in the figure. Under conditions of local thermodynamic equilibrium, the "main" line is intrinsically stronger than the satellite line by a factor of $\sim 16$. However, from the $\mathrm{NH}_{3}(2,2)$ spectrum, we see that the peak intensities of the "main" and "satellite" lines are roughly equal. This is an indication of high opacity in this region. The mean $\mathrm{NH}_{3}(2,2)$ emission in the main component is shown in Fig. 4 (top). The radio continuum emission at $1.3 \mathrm{~cm}$ is shown as contours. The map obtained by averaging the emission in the channels comprising the satellite lines is shown in Fig. 4 (bottom). In both cases, it can be seen that the $\mathrm{NH}_{3}$ emission peaks at a location that is slightly north-west of the radio continuum emission.

From the integrated spectrum, we observe that the "main" component is asymmetrical suggesting either self-absorption or presence of other components along the line-of-sight. Most of the emission is at velocities greater than the systemic velocity of the source, $V_{\mathrm{LSR}} \sim 111 \mathrm{~km} \mathrm{~s}^{-1}$. An average optical depth is determined from the ratio of the maximum intensities observed for the main and the satellite components. In this case, since the main line is non-Gaussian and looks selfabsorbed, such a ratio provides us with an upper limit to the optical depth. For the observed line ratio of 1.5, the optical depth is hence $<18$. Beltrán et al. (2005) have determined gas temperature of $\sim 100 \mathrm{~K}$ for G24 A2. For this temperature and the deduced opacity, under the assumption of local thermodynamic equilibrium, the column density for the $(J, K)=(2,2)$ level is calculated $N_{2,2}<1.1 \times 10^{17} \mathrm{~cm}^{-2}$. For a $\mathrm{NH}_{3}$ partition 


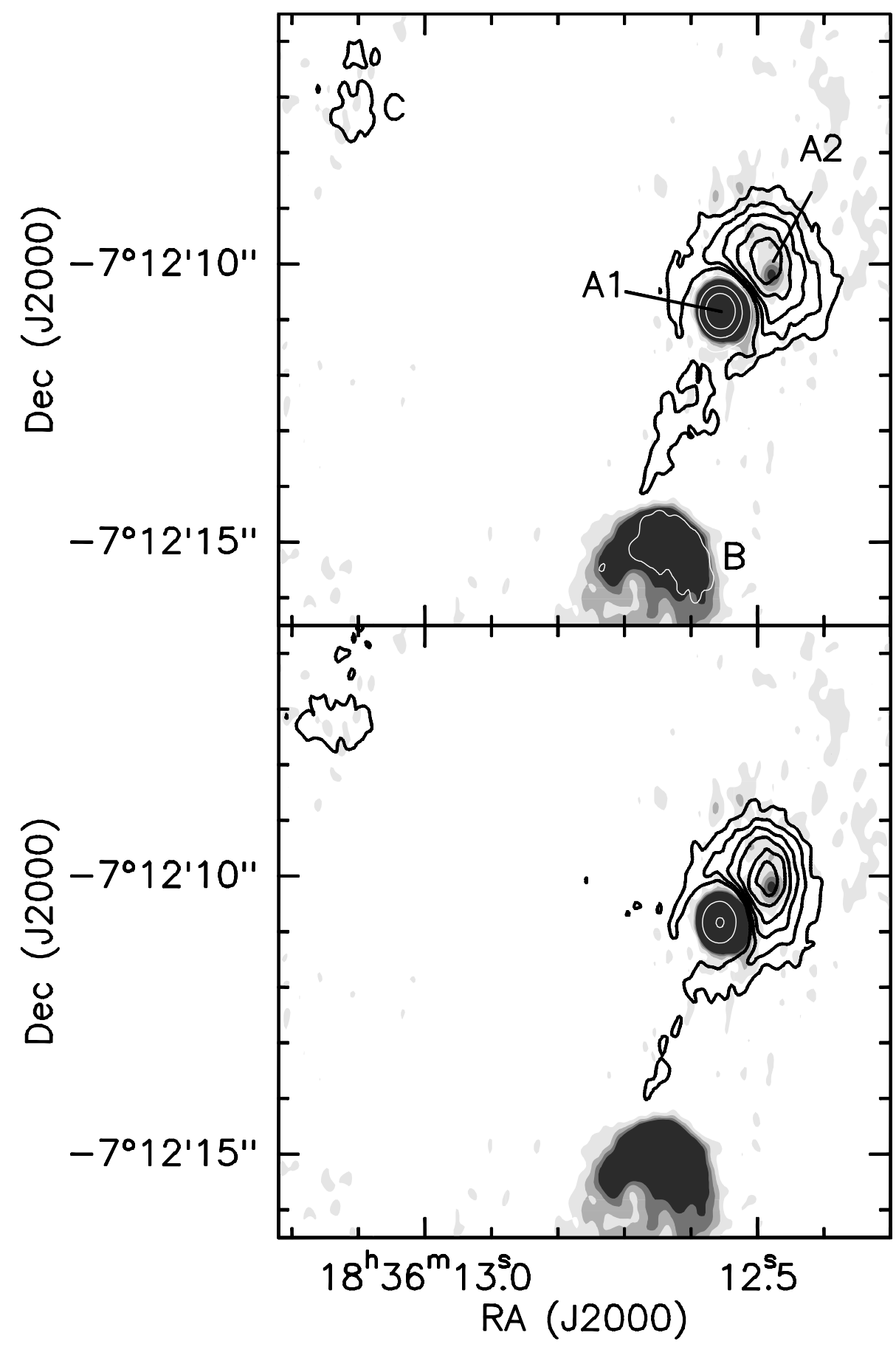

Fig. 3. The grayscale image is the radio continuum emission at $1.3 \mathrm{~cm}$ from the G24 region. The image has been saturated for better viewing of emission from G24 A2. The grayscale levels range from 0.2 to $0.8 \mathrm{mJy} /$ beam in steps of $0.2 \mathrm{mJy} /$ beam where beam is circular of size $\sim 0$ ' $^{\prime}$. (Top) The black contours represent the average $\mathrm{NH}_{3}(2,2)$ emission from the main component while the white contours represent absorption. The contour levels (black line) are 1.6 to $12.8 \mathrm{mJy} /$ beam by $1.6 \mathrm{mJy} / \mathrm{beam}$ and (white line) -1.6 to $-30 \mathrm{mJy} / \mathrm{beam}$ by $-10 \mathrm{mJy} /$ beam where the beam is $0.27 \times$ 0 ' 23 . The sources A1, A2, B and C are marked. (Bottom) Contours representing average emission (black line) and absorption (white line) in the $\mathrm{NH}_{3}(2,2)$ satellite. The contour levels (black line) are 0.18 to $2.8 \mathrm{mJy} / \mathrm{beam}$ by $0.2 \mathrm{mJy} / \mathrm{beam}$ and (white line) -1.6 to $-4.5 \mathrm{mJy} /$ beam by $-1.6 \mathrm{mJy} / \mathrm{beam}$. function $Q(T)=0.0277 T^{3 / 4}(\mathrm{~K})$ and a statistical weight $g_{2,2}=$ 5 , we get a total column density $N\left(\mathrm{NH}_{3}\right)<1.2 \times 10^{18} \mathrm{~cm}^{-2}$.

\subsection{Spectral energy distribution}

The fluxes at the near- and mid-infrared wavelengths for G24 A2 from the IRAC-GLIMPSE as well as VLT-VISIR have been used in constructing the spectral energy distribution (SED). G24 A2 has not been detected in the near-infrared in the Two Micron All Sky Survey (2MASS). The $1.4 \mathrm{~mm}$ continuum flux (measured by Beltrán et al. 2004) has also been considered. The SED has been dereddened by 14 mag of visual extinction $\left(1.8 \mathrm{mag} \mathrm{kpc}^{-1}\right)$ corresponding to the extinction due to the interstellar medium (Whittet 1992) for a distance of $7.7 \mathrm{kpc}$. For the Spitzer-IRAC bands, we have used the average extinction values obtained by Indebetouw et al. (2005). The reddening law from Rieke \& Lebofsky (1985) has been applied to the VISIR $11.9 \mu \mathrm{m}$ band. At 18.7 and $24 \mu \mathrm{m}$, we have used extrapolated extinction values. The dereddened fluxes are listed in the last column Table 1. Figure 6 shows the spectral energy distribution of G24 A2. We see that the SED rises steeply with increasing wavelength till $5.8 \mu \mathrm{m}$ and thereafter shows a drop. By linearly interpolating between the observed fluxes (near- and mid-infrared as well as mm continuum) on the logarithmic scale (i.e. power-law interpolation on linear scale), one obtains a very conservative lower limit of $370 L_{\odot}$ to the bolometric luminosity of G24 A2. A better estimate may be obtained by fitting models of embedded young stellar objects to the SED. In the following subsection, we explore such models. 


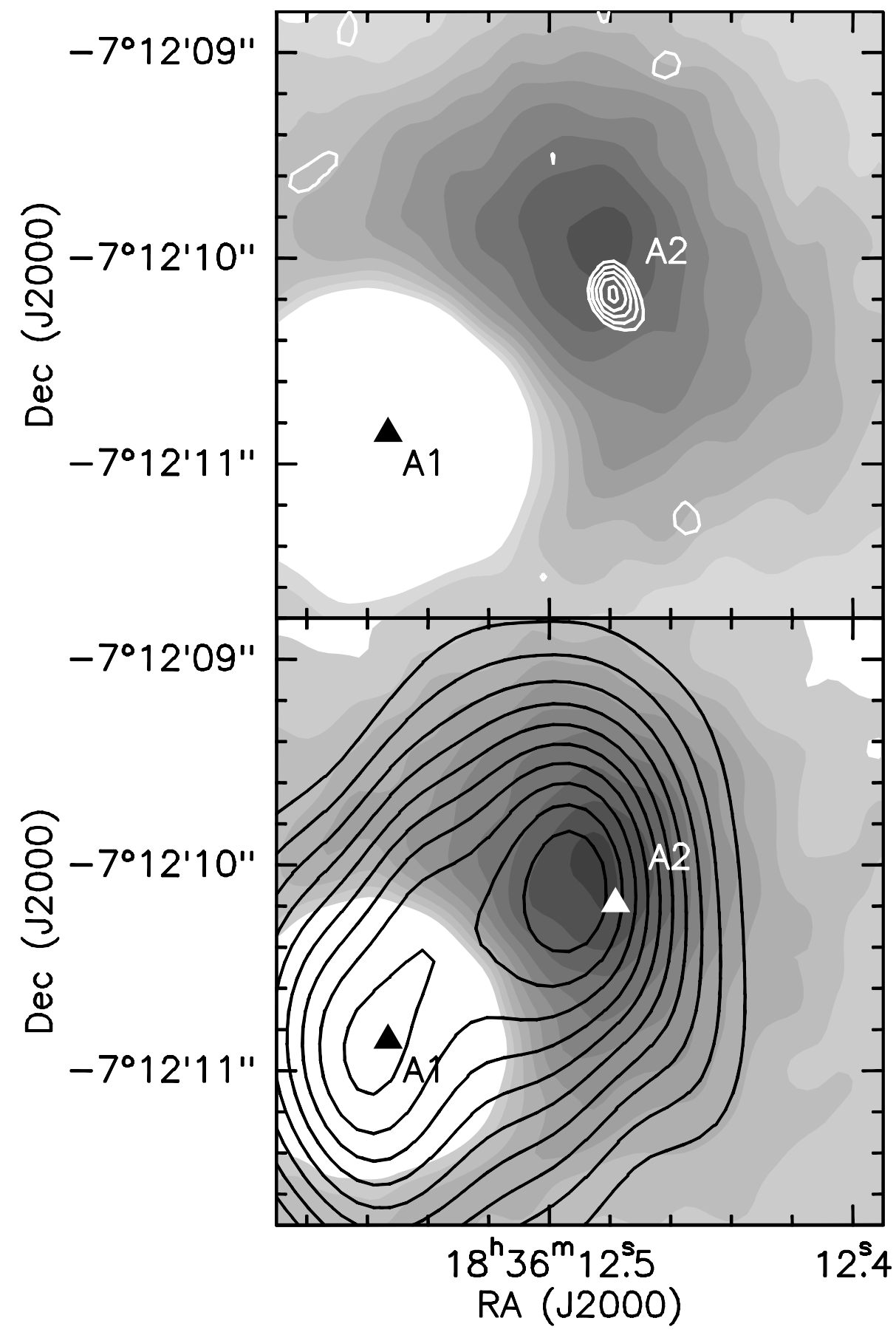

Fig. 4. (Top) The image represents the $\mathrm{NH}_{3}$ emission averaged over the main component reconstructed with a circular cleam beam of $0 . ' 8$. The grayscale levels range from 0 (white) to $9.7 \mathrm{mJy} /$ beam (deep gray) in steps of $1 \mathrm{mJy} / \mathrm{beam}$. The white contours depict the $1.3 \mathrm{~cm}$ radio continuum emission with contour levels ranging from 0.35 to $1 \mathrm{mJy} /$ beam in steps of $0.1 \mathrm{mJy} / \mathrm{beam}$, where the beam is $0.27 \times 0$ ' 23 . The triangle represents the peak of radio emission from A1 at $1.3 \mathrm{~cm}$. (Bottom) The black contours show the $\mathrm{CH}_{3} \mathrm{CN}(12-11)$ emission for the $K=3$ component overlaid on the $\mathrm{NH}_{3}$ emission averaged over the satellite components. The grayscale levels of the $\mathrm{NH}_{3}$ emission range from 0 (white) to $1.45 \mathrm{mJy} /$ beam (deep gray) in steps of $0.14 \mathrm{mJy} / \mathrm{beam}$. The contour levels of the $\mathrm{CH}_{3} \mathrm{CN}$ emission are 0.25 to $1.15 \mathrm{Jy} / \mathrm{beam}$ in steps of $0.1 \mathrm{Jy} /$ beam, where the beam is 1 '. $2 \times 0$.' 5 . The triangles mark the locations of the peak of radio emission at $1.3 \mathrm{~cm}$.

\subsubsection{RWIW models}

In order to get a more realistic estimate of the luminosity of G24 A2, we have used the models of Robitaille et al. (2007, hereafter RWIW). These authors have computed a large set of radiation transfer models and obtained the SEDs for a reasonably large parameter space. These SEDs can be fitted to multiwavelength observational data of single sources to constrain their physical parameters. The best constraints are obtained when the near-, mid- and far-infrared as well as (sub)millimetre data are included.

We have used the SED of G24 A2 with the near- and midinfrared (dereddened by 14 mag of visual extinction, listed in Table 1) along-with the millimetre data to fit the RWIW models. We have compared the observed fluxes with the model fluxes in various wavebands and the models are selected based on the minimum chi-square method. For the fluxes at 12 and $18 \mu \mathrm{m}$, we have compared the observed fluxes with the computed monochromatic model fluxes (provided by Robitaille). Figure 6 shows the SEDs of ten RWIW models selected. The near-infrared upper limits from 2MASS are also shown for comparison. We emphasize that we have not used any rigorous selection criteria in selecting these models. The models are those whose fluxes reasonably match the observed fluxes. All the selected models require a massive young protostar (7.6-27.4 $\left.M_{\odot}\right)$ as the central object, with an age ranging from 1200 to $4200 \mathrm{yrs}$ and temperatures of the order of $\sim 4200 \mathrm{~K}$. The total luminosity (central object + envelope and/or disk) computed by RWIW is $1.1-24 \times 10^{3} L_{\odot}$. All the models have relatively high accretion rates $\left(1-7 \times 10^{-3} M_{\odot} \mathrm{yr}^{-1}\right)$. These models with envelopes either 


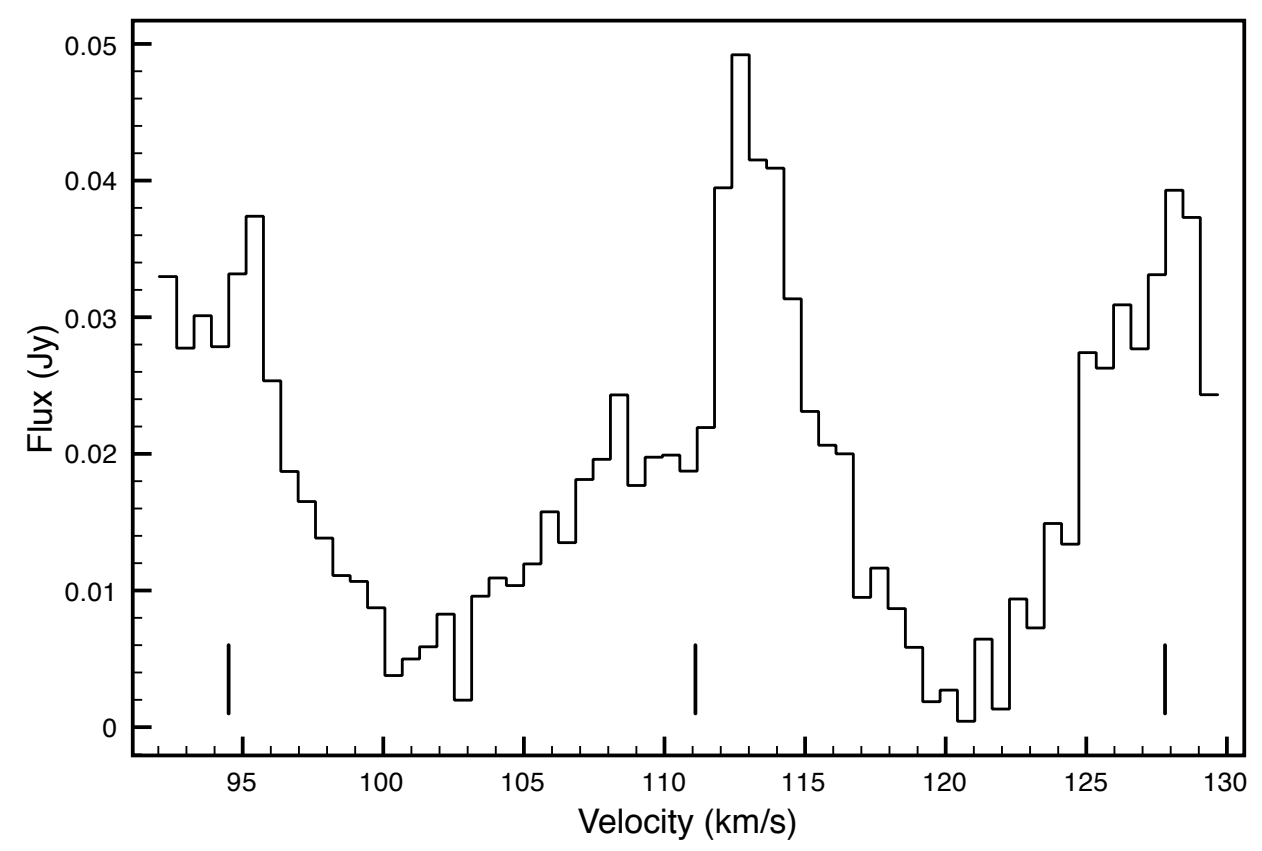

Fig. 5. Spectrum of $\mathrm{NH}_{3}(2,2)$ averaged over the emission from the entire G24 A2 region. The main component (centre) can be seen along with the two inner satellites on either side. The centres of the lines corresponding to the rest frequency of the $\mathrm{NH}_{3}$ main line and satellites are marked near the base for a $V_{\mathrm{LSR}}=111 \mathrm{~km} \mathrm{~s}^{-1}$.

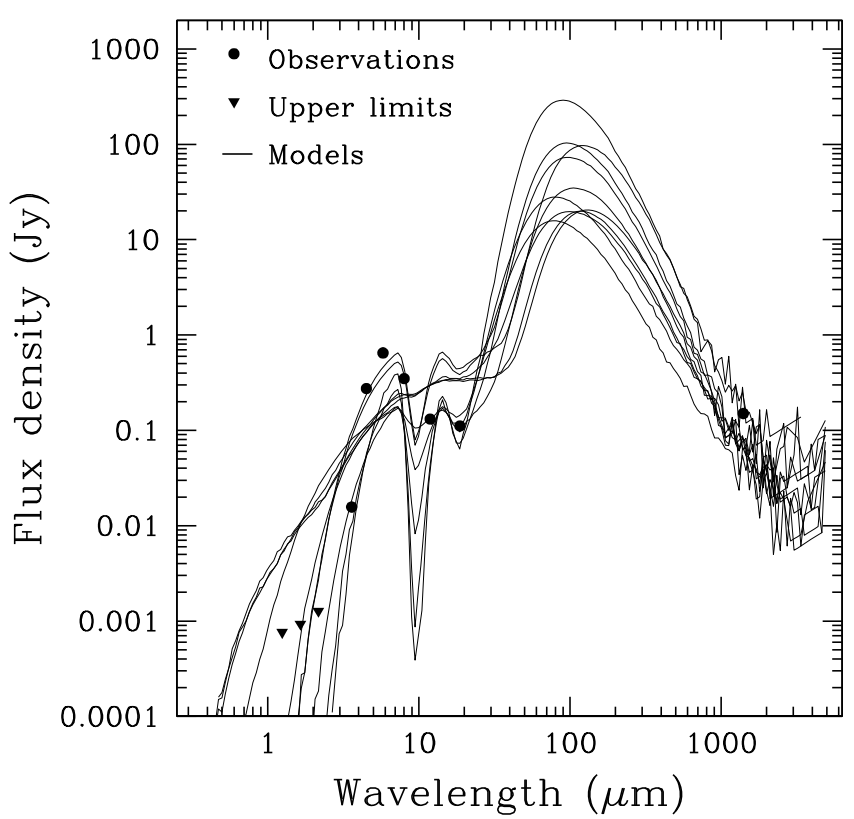

Fig. 6. The spectral energy distribution of G24 A2 constructed from observations (filled circles) using Spitzer-GLIMPSE bands, VLT-VISIR and PdBI 1.4 millimetre data. The infrared fluxes have been dereddened by 14 mag of visual extinction. The solid triangles represent the upper limits to the fluxes using the 2MASS data. The solid lines represent the best-fit Robitaille et al. (2007) models.

have no disks or very little mass $\left(0.2 M_{\odot}\right)$ in disks. However, all these models have an inclination of $\sim 20^{\circ}$ where $90^{\circ}$ represents the edge-on case. This inclination angle decides the direction of the cavity (cavity semi-opening angle $2-4^{\circ}$ ) and hence of the outflow. The parameters obtained from RWIW models for G24 A2 show that it represents a massive embedded young protostellar object. Although this result seems reasonable, it should be noted that these models are valid for isolated objects and the effect of close neighbours on their environment has not been considered. Given the presence of multiple young stellar objects in the G24 region, it is difficult to evalutate the reliability of the parameters obtained from the RWIW fits to the G24 A2 SED. In addition, it is to be noted that the flux at the longer wavelength $(5.8$ and $8.0 \mu \mathrm{m})$ IRAC bands could have contributions from Polycyclic Aromatic Hydrocarbons (PAH) bands at 6.2, 7.7 and $8.6 \mu \mathrm{m}$. Nevertheless, these models provide qualitative information about the nature of the object and allow us to set constraints on its luminosity.

\section{Nature of G24 A2}

\subsection{Clues from the continuum emission}

An investigation of the IRAC images shows that the position of the peak emission agrees within 1 pixel $\left(1\right.$ pixel $\left.=1^{\prime \prime} .2\right)$ but there is no trend of a change in position with wavelength. The GLIMPSE catalog lists the position of this source at $\alpha(\mathrm{J} 2000)=$ $18^{\mathrm{h}} 36^{\mathrm{m}} 12^{\mathrm{s}} 484, \delta(\mathrm{J} 2000)=-07^{\circ} 12^{\prime} 10^{\prime} \cdot 15$. This coincides with the position of the of the radio continuum emission within $0 .^{\prime} 1$. So, for our purposes here, we consider that the mid-infrared emission is associated with an embedded young stellar object in G24 A2. This emission could have contributions from (a) thermal emission from warm dust in the circumstellar envelope and/or massive toroid (Beltrán et al. 2004) heated by direct stellar radiation; (b) heating of dust due to Lyman- $\alpha$ photons resonantly scattering in the ionised region (Hoare et al. 2007); and (c) scattered light from dust associated with a jet or outflow (De Buizer et al. 2005). It is difficult to assign a hot-dust temperature based on these mid-infrared colours as the mid-infrared emission is likely to have contribution from small grains (Puget \& Leger 1989).

The radio continuum emission associated with G24 A2 is unresolved as well as weak (Sect. 3.2). An investigation of the high angular resolution maps ( 60 mas) of G24 A obtained by Beltrán et al. (2007) at $1.3 \mathrm{~cm}$ and $7 \mathrm{~mm}$ does not show the 


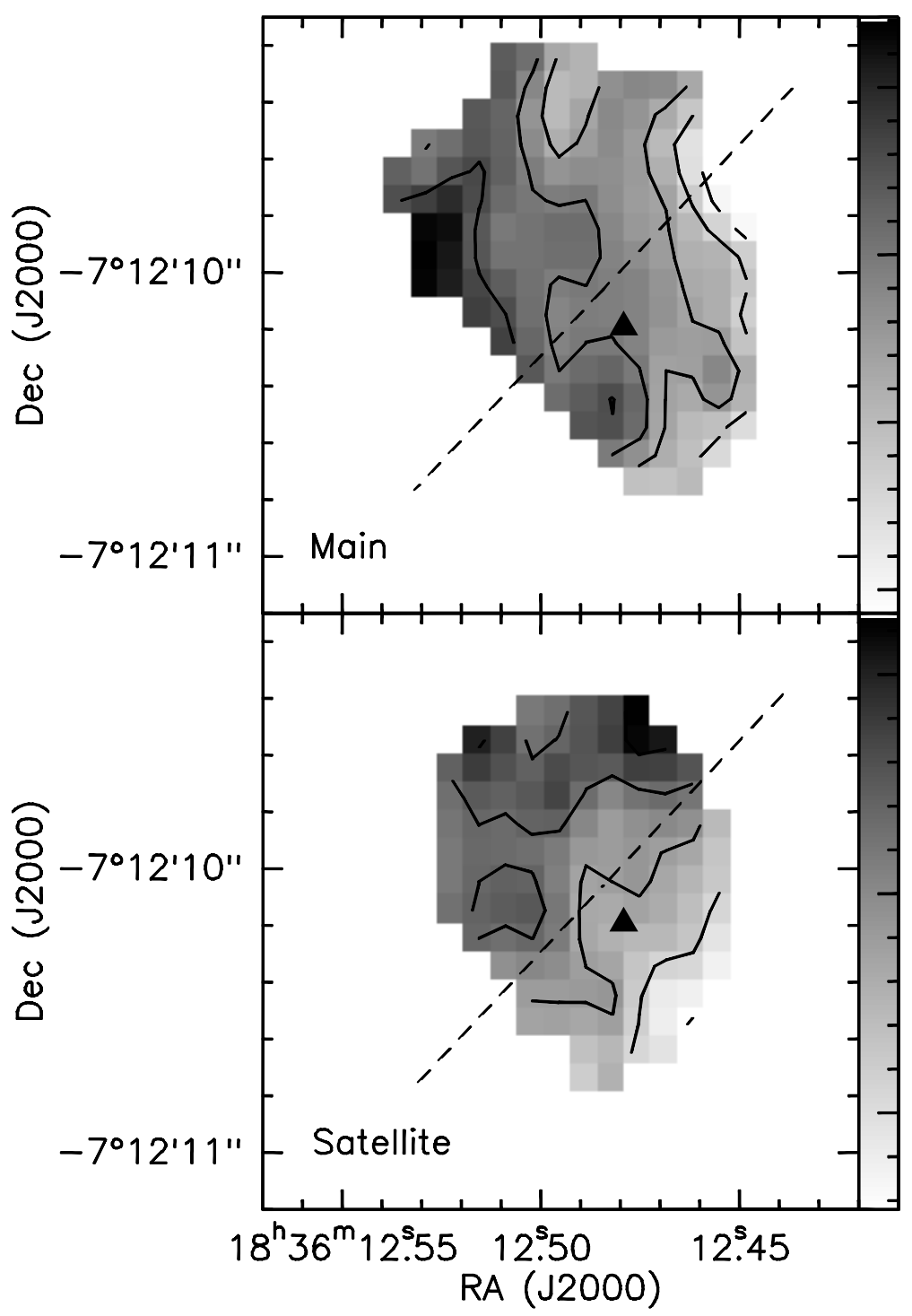

112

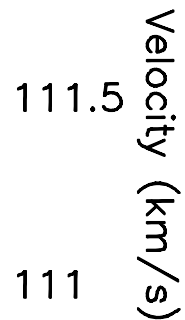

110.5

111.5

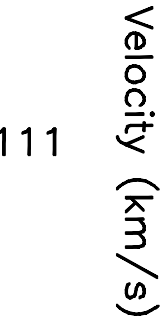

110.5

Fig. 7. The velocity distribution towards G24 A2 from the (top) main component and (bottom) satellite of $\mathrm{NH}_{3}(2,2)$ transition. The triangle marks the location of the radio continuum emission at $1.3 \mathrm{~cm}$. The dashed line represents the direction parallel to the ${ }^{12} \mathrm{CO}$ outflow axis (Furuya et al. 2002). The contour levels range from 110.3 to $111.8 \mathrm{~km} \mathrm{~s}^{-1}$ in steps of $0.3 \mathrm{~km} \mathrm{~s}^{-1}$.

presence of any emission at the location of $\mathrm{A} 2$ whereas pointlike emission was detected with a resolution of 0.25 at $1.3 \mathrm{~cm}$ and 0.48 at $7 \mathrm{~mm}$. This indicates that the radio continuum emission is from a region larger than 60 mas and the emission is too weak to be detected at such high angular resolutions. This enables us to obtain limits on the diameter $D$ of this source: $1000 \mathrm{AU}<D<2000 \mathrm{AU}$. Based on the fact that the emission is resolved out at $1.3 \mathrm{~cm}$ using a $\sim 60$ mas beam, we have used the upper limit to the flux ( $3-\sigma$ at $1.3 \mathrm{~cm}: 0.25 \mathrm{mJy} /$ beam, beam $\sim 0{ }^{\prime} 95 \times 0$ 0.56; Beltrán et al. 2007) to determine the lower limit to the diameter. The upper limit to the size of the radio emitting region of $2000 \mathrm{AU}$ has been estimated using the fact that the emission is unresolved in a 0 !' 25 beam. It is unlikely that this radio continuum emission is optically thick free-free emission as the measured brightness temperature is $30 \mathrm{~K}<T_{\mathrm{B}}<110 \mathrm{~K}$. For optically thick emission, $T_{\mathrm{B}}$ is expected to be at least $\sim 10^{3} \mathrm{~K}$. In the present case, had the emission been optically thick, it should have been detected in the high angular resolution ( $60 \mathrm{mas})$ images. The spectral index is determined to be $1.9 \pm 0.4$ from the detections at $1.3 \mathrm{~cm}$ and $7 \mathrm{~mm}$. However, it is difficult to draw conclusions on the optical depth based on the spectral index alone as there could be contribution from dust emission at $7 \mathrm{~mm}$. Recent continuum measurements at $3.6 \mathrm{~cm}$ with the VLA have led to the detection of G24 A2 with a flux of 0.3 mJy (Beltrán et al., personal communication). This gives a spectral index of $\sim 1$ between 1.3 and $3.6 \mathrm{~cm}$ which suggests that the emission could be from a jet (see e.g. Anglada 1996). An H II region with a density gradient can also explain this spectral index. However, further sensitive measurements at different wavelengths are required in order to draw conclusions about the nature of this radio source.

\subsection{The $\mathrm{NH}_{3}$ line emission}

The $\mathrm{NH}_{3}(2,2)$ emission observed towards $\mathrm{G} 24 \mathrm{~A} 2$ shows a peak which is $\sim 0^{\prime} \cdot 3$ to the north-west of the radio continuum emission as seen in Fig. 4. Although small, this difference in peak emission cannot be overlooked since the $\mathrm{NH}_{3}$ line and the $1.3 \mathrm{~cm}$ radio continuum have been observed simultaneously. Figure 4 also shows the $\mathrm{CH}_{3} \mathrm{CN}(12-11)$ emission overlaid as contours for the $K=3$ component.

We need to understand the spectral signatures of the $\mathrm{NH}_{3}$ emission from different parts of the region associated with G24 A2. Combining the satellite lines on either side of the main component to obtain the full profile of a single satellite line, we have been able to obtain the velocity distribution traced by the satellite emission. Figure 7 compares the distribution of the velocity around G24 A2 for the $\mathrm{NH}_{3}$ main (top) and satellite 


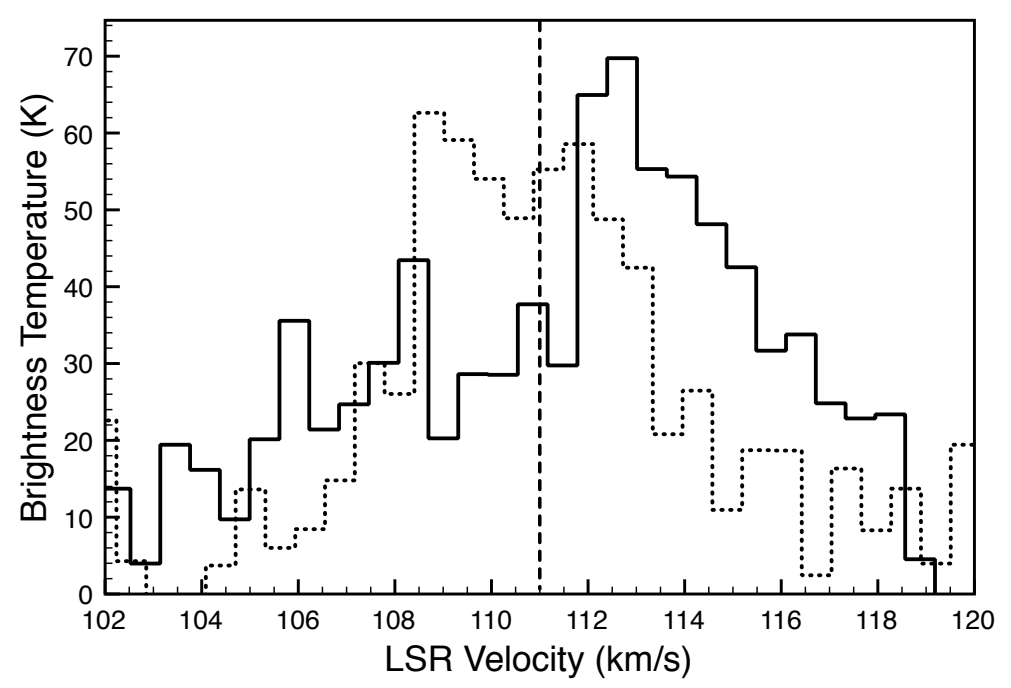

Fig. 8. Spectrum of $\mathrm{NH}_{3}(2,2)$ main component (solid line) and satellite (dotted line) towards the central exciting object (location of the peak of the radio continuum emission). The vertical dashed line represents the systemic velocity.

(bottom) lines. The direction parallel to the ${ }^{12} \mathrm{CO}$ outflow (Furuya et al. 2002) is shown as a dashed line while the triangle marks the location of the $1.3 \mathrm{~cm}$ radio continuum emission. These velocity maps have been obtained as the first moment (i.e. intensity weighted velocity of the spectal line) of the main and satelline components. It is interesting to note that the velocity distribution of the satellite line is found to be similar to that obtained using the $\mathrm{CH}_{3} \mathrm{CN}$ (12-11) line by Beltrán et al. (2004). This can be interpreted as rotation of G24 A2 as suggested by Beltrán et al. (2004). On the other hand, the velocity distribution of the $\mathrm{NH}_{3}(2,2)$ main component shows a similar trend but oriented E-W rather than NE-SW like in the case of the satellite and $\mathrm{CH}_{3} \mathrm{CN}$ lines. We believe this is due to the non-Gaussian shape of the main line. Figure 8 shows the spectra of the main (solid line) and the satellite (dotted) line of $\mathrm{NH}_{3}(2,2)$ towards the position of the peak of the radio continuum emission which we assume to be the location of the central exciting star. The vertical dashed line represents the systemic velocity. From this figure, an asymmetry can be clearly perceived in the main component towards the red side. On the other hand, the satellite line shows a more symmetric behaviour and is stronger than the main component at the systemic velocity. A comparison of the main and satellite components clearly shows that the former is selfabsorbed and the fact that the dip is blue-shifted indicates that this self-absorption occurs in an expanding envelope. In the following, using all the previous findings, we attempt to understand the nature of G24 A2.

\subsection{A model for an expanding and rotating toroid}

From the velocity distribution in Fig. 7, the signature of rotation is clearly discernible. We propose that in addition to rotation, there is expansion of gas around A2 based on the spectral signature seen towards the centre of G24 A2. In order to decouple these two motions, we look at the velocity distribution at the boundary and the centre of the emission towards G24 A2. It is expected that while the effect of rotation is maximum along the edges, the effect of systematic motions like infall or expansion are best viewed towards the centre. For the interpretation of our results, we consider the following facts: (a) the velocity gradient is observed in the satellite component along a direction perpendicular to the ${ }^{12} \mathrm{CO}$ outflow revealed by Furuya et al. (2002) and (b) the $\mathrm{NH}_{3}(2,2)$ spectrum towards the location of G24 A2 (identified as the peak of radio continuum emission) shows a distinct red asymmetry. The latter can be distinctly viewed through the position-velocity (PV) plots of $\mathrm{NH}_{3}(2,2)$ in Fig. 9 with the position cut made along the plane of the toroid $\left(\mathrm{PA}=-135^{\circ}\right)$. While the PV plot of the satellite component shows a more symmetric behaviour than that of the main line, the main line shows a peak at velocities larger than the systemic velocity which we interpret as due to self-absorption. While the velocity gradient (also seen in the $\mathrm{CH}_{3} \mathrm{CN}(12-11)$ transition) has been interpreted to be due to rotational motion in the toroid, we propose that in addition, there is expansion of gas around A2. We used a simple model of an edge-on disk to ascertain whether the observed PV plots of $\mathrm{CH}_{3} \mathrm{CN}(12-11), \mathrm{NH}_{3}(2,2)$ main, and satellite components can be consistently explained by a motion which is a combination of rotation and expansion. We assume the case of an edge-on disk undergoing solid-body rotation and expansion with $v \propto R$. The details of the model are given in Appendix A. The input parameters of the models are the inner and outer radii of the toroid $\left(R_{\mathrm{i}}, R_{\mathrm{O}}\right)$, a parameter related to the column density at the peak of the emission $\left(N_{\text {peak }}\right)$, the intrinsic line width $(\Delta v)$, the power-law index of the temperature $(q)$ as well as the rotation and expansion velocities at the outer edge of the toroid $\left(v_{\theta 0}\right.$, $\left.v_{\text {ro }}\right)$. The model PV plots were computed for the $\mathrm{CH}_{3} \mathrm{CN}(12-11)$ $K=3$ and $\mathrm{NH}_{3}(2,2)$ main and satellite emission and convolved with the respective beams for a meaningful comparison with the observations. The initial guesses for few of the input parameters have been obtained from the observations. In fact, the rotation velocity is obtained from the velocity distribution plot (see Fig. 7) while an indicator of the expansion velocity is the difference in peak intensities of the spectral line profiles of $\mathrm{NH}_{3}(2,2)$ main and satellite components. The other parameters like $\Delta v, \mathrm{q}$, and $N_{\text {peak }}$ have been obtained by changing their values in order to obtain good fits. The models that have been selected are those that suitably fit the observed data in intensity as well as in morphology and are as self-consistent as possible among the three sets of parameters apart from the column densities which may obviously be different for the two molecules.

The observed and synthesized PV diagrams are shown in Fig. 9. The positional cut is made along the plane of the disk. In all the panels, the grayscale represents the positionvelocity plots of the measured emission from the $\mathrm{NH}_{3}(2,2)$ or 


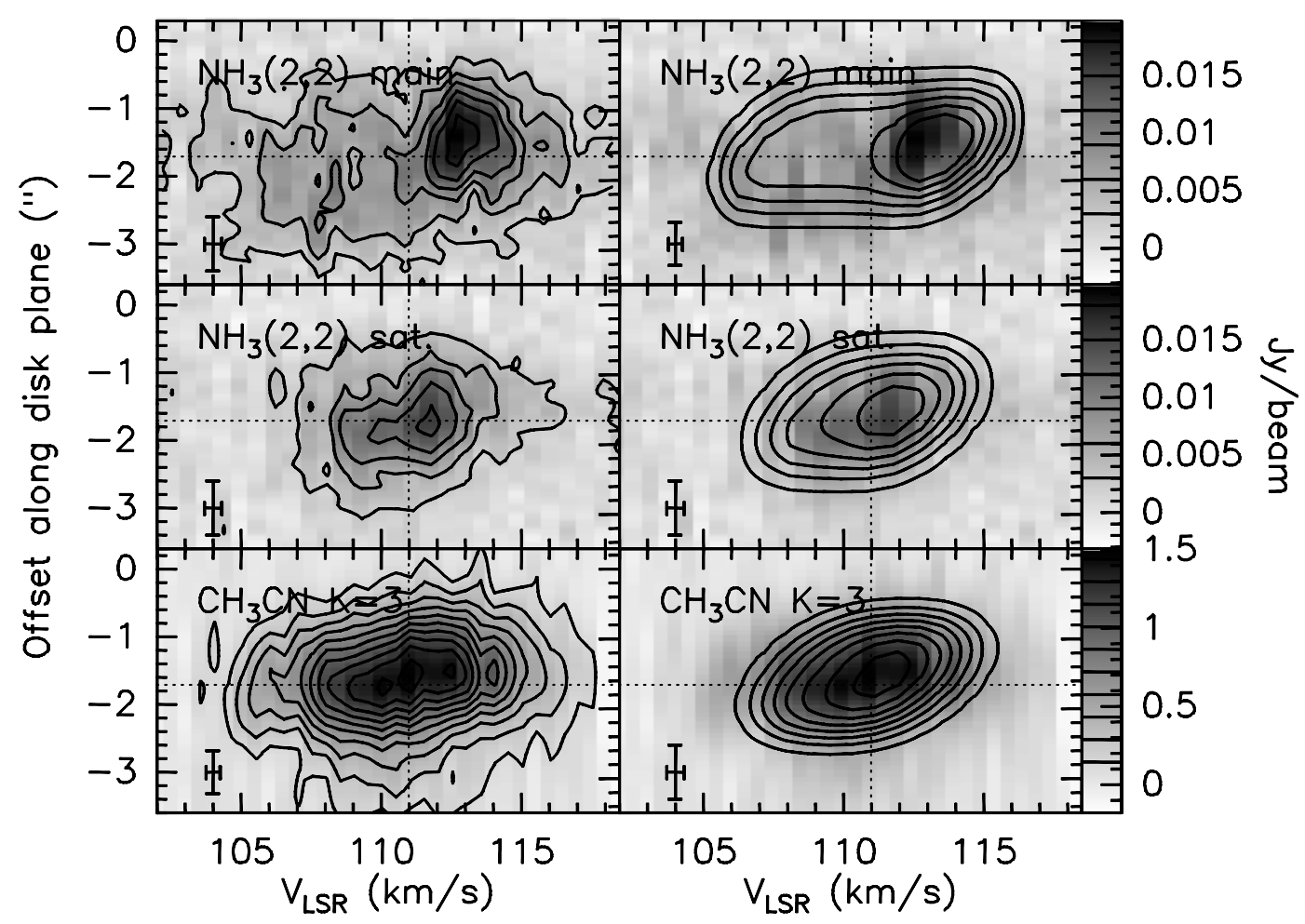

Fig. 9. Position-velocity plot of $\mathrm{NH}_{3}(2,2)$ main component (top panel), satellite (middle panel) and $\mathrm{CH}_{3} \mathrm{CN}(12-11) \mathrm{K}=3$ emission (lower panel) in G24 A2. The positional cut is made along the plane of the disk. In all the panels, the grayscale represents the position-velocity plots of the measured emission from $\mathrm{NH}_{3}(2,2)$ or $\mathrm{CH}_{3} \mathrm{CN}(12-11) K=3$ components. (Left) Contours correspond to the measured emission, which start at 3- $\sigma$ level and increase by 3- $\sigma$. (Right) Contours levels correspond to the synthetic emission from the model described in Sect. 4.3. The contour levels are the same as that used in the left panel. The error bars denote the angular and spectral resolution. The vertical and horizontal dotted lines represent the systemic velocity and the position of the $\mathrm{CH}_{3} \mathrm{CN}$ peak, respectively.

Table 2. Model parameters for G24.78-0.08 A2. The details of the model can be found in Sect. 4.3 as well as in Appendix A.

\begin{tabular}{ccccccccccc}
\hline \hline Molecule & \multicolumn{2}{c}{$R_{\mathrm{i}}$} & \multicolumn{2}{c}{$R_{\mathrm{o}}$} & $\begin{array}{c}T_{\mathrm{o}} \\
\left({ }^{\prime \prime}\right)\end{array}$ & $\begin{array}{c}N_{\text {peak }} \\
(\mathrm{AU})\end{array}$ & $\begin{array}{c}\Delta v \\
(\mathrm{~K})\end{array}$ & $\begin{array}{c}v_{\theta \mathrm{o}} \\
\left(\mathrm{km} \mathrm{s}^{-1}\right)\end{array}$ & $\begin{array}{c}v_{\text {ro }} \\
\left(\mathrm{km} \mathrm{s}^{-1}\right)\end{array}$ & $q$ \\
$\left(\mathrm{~km} \mathrm{~s}^{-1}\right)$ & \\
\hline $\mathrm{CH}_{3} \mathrm{CN}$ & 0.1 & 770 & 0.9 & 6900 & 65 & $1.4 \times 10^{16}$ & 2.7 & 1.6 & 2.4 & -0.75 \\
$\mathrm{NH}_{3}$ & 0.1 & 770 & 0.9 & 6900 & 50 & $9.0 \times 10^{17}$ & 2.6 & 1.5 & 2.1 & -0.6 \\
\hline
\end{tabular}

$\mathrm{CH}_{3} \mathrm{CN}(12-11) \mathrm{K}=3$ lines. The model parameters are listed in Table 2. We find that constant density models fit the data reasonably well. The temperature gradients, $q \sim-0.6$ and -0.75 are similar to that obtained by accretion disk theory for geometrically thin disks $\left(T \propto R^{-0.75}\right)$ but we note that this not a unique interpretation. The values of the inner and outer radii are $0 .^{\prime} 1$ (770 AU) and $0 .^{\prime} 9$ (6900 AU), respectively. The rotation and expansion velocities correspond to $\sim 1.5$ and $\sim 2.1 \mathrm{~km} \mathrm{~s}^{-1}$ and these are roughly the values obtained from the observations. The column density at the peak of the $\mathrm{CH}_{3} \mathrm{CN}$ emission obtained from the model $\left(1.4 \times 10^{16} \mathrm{~cm}^{-2}\right)$ agrees with that measured by Beltrán et al. (2005), within a factor of $\sim 3$. The latter, $N\left(\mathrm{CH}_{3} \mathrm{CN}\right)=4 \times 10^{16} \mathrm{~cm}^{-2}$, has been derived using the rotation diagram method. The $\mathrm{NH}_{3}$ column density from the model $\left(9 \times 10^{17} \mathrm{~cm}^{-2}\right)$ is consistent with that obtained from the observations $N\left(\mathrm{NH}_{3}\right)<1.2 \times 10^{18}$ (Sect. 3.3). The intrinsic line width (due to thermal effects and microturbulence) that fits the data is $\sim 2.6 \mathrm{~km} \mathrm{~s}^{-1}$. Figure 9 (right) shows that the models with the above mentioned parameters fit all the available data well. We stress that the only purpose of our model fit is to demonstrate that one of the plausible explanations of the observed distribution is that it is due to a motion that combines rotation and expansion.

\subsection{Possible scenarios of G24 A2}

In order to comprehend the nature of this expansion close to G24 A2, two scenarios have been explored. In both scenarios, we believe that the expansion is due to the outflow detected in G24 A by Furuya et al. (2002). Either A1 or A 2 could be powering the outflow as both toroids in A1 and A2 are perpendicular to the outflow axis. Here we show that both cases can qualitatively explain the spectra as well as the velocity distribution of G24 A2 and G24 A1.

In the first case, $\mathrm{A} 2$ is powering the outflow. The expanding gas can produce the red asymmetry seen towards the centre in the main $\mathrm{NH}_{3}$ component as shown in Sect. 4.2.1. The absence of such an asymmetry in the satellite line is explained because this emission is more optically thin. This expansion velocity field when combined with the one due to rotation, will explain the velocity distribution obtained by us for G24 A2. Figure 10 (left) shows a schematic of this scenario. For the sake of completeness, we note that the broad blue-shifted wing seen in absorption of the $\mathrm{NH}_{3}(2,2)$ line towards G24 A1 (see Fig. 2 of Beltrán et al. 2006) cannot be due to the blue lobe of the outflow, because in this scenario such a lobe does not lie between A1 and 

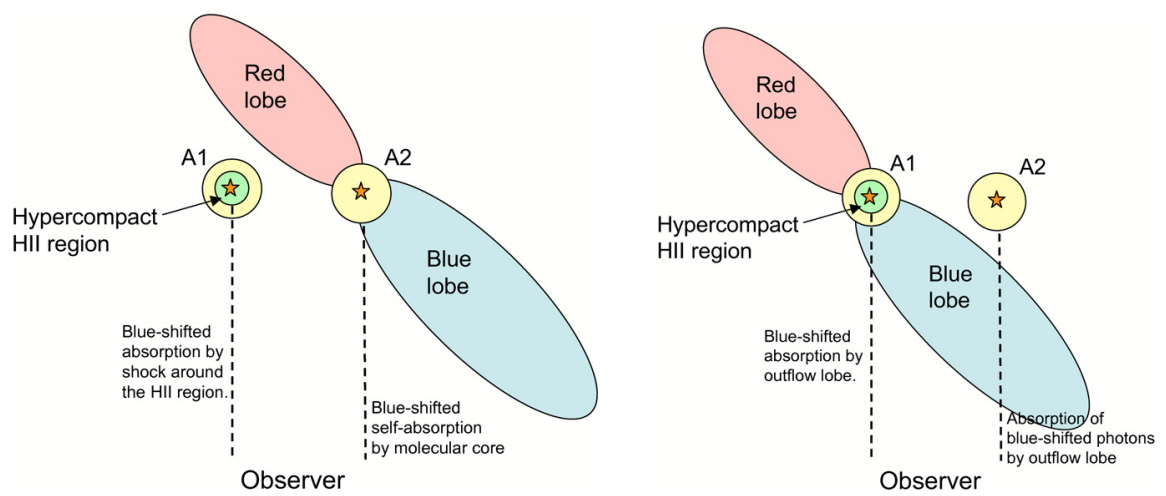

Fig. 10. Schematic picture of G24 A showing two possible scenarios: (left) A2 is the origin of the outflow, and (right) A1 is powering the outflow. The larger circles around $\mathrm{A} 1$ and $\mathrm{A} 2$ represent the hot molecular cores, while the smaller circle around A1 represents the hypercompact $\mathrm{H}$ II region. Here, the dashed lines are parallel to the line-of-sight to the observer who is located at the bottom of the figure. the observer. A possible explanation is that the blue-shifted absorption in G24 A1 is due to the compressed layer of gas in the post-shock at the border of the hypercompact H II region imaged by Beltrán et al. (2007).

In the second scenario, we assume that A1 is the driver of the outflow. In such a situation, the blue lobe of the outflow would pass in front of A2. The schematic picture of this scenario is depicted in Fig. 10 (right). If the $\mathrm{NH}_{3}$ column density in the blue lobe is sufficiently high, the blue part of the $\mathrm{NH}_{3}$ line emitted by $\mathrm{A} 2$ will be absorbed resulting in a skewed profile with the intensity of the red part larger than that corresponding to the blue. This also explains the broad blue-shifted absorption in the main $\mathrm{NH}_{3}(2,2)$ line detected towards A1 (Beltrán et al. 2006) as due to absorption in the densest part of the blue lobe towards the A1 H II region.

Although we have considered two simple scenarios with either A2 or A1 being the source of the outflow, we believe the former to be more plausible. This is because the larger $\mathrm{NH}_{3}(2,2)$ optical depth needed in the latter would imply very large $\mathrm{NH}_{3}$ column density in the outflow lobe. Further, the emission from the $\mathrm{NH}_{3}(2,2)$ satellite (as well as $\mathrm{CH}_{3} \mathrm{CN}$ ) is from denser gas closer to the central object, so that it seems unlikely that the $\mathrm{NH}_{3}$ main line emission originates from a different region.

Finally, we note that the proposed scenarios do not rule out the possibility that two parallel outflows (one from A1, the other from A2) exist.

\section{Comparison between G24 A1 and G24 A2}

The properties of the subcores of G24 A, viz. G24 A1 and G24 A2, are compared in this section. While on the one hand, $\mathrm{A} 1$ and A2 show many similar properties, there are some differences that may represent clues to unveiling their nature. Our goal here is to draw conclusions based on the similarities and differences of these two neighbouring cores. The properties that are similar include: (1) mass of the cores (130 and $80 M_{\odot}$ for A1 and A2 respectively, based on Beltrán et al. 2004); (2) the core temperatures $(\sim 100 \mathrm{~K})$ and; (3) similar rotation axes as shown by the velocity gradients seen in $\mathrm{CH}_{3} \mathrm{CN}(12-11)$ and $\mathrm{NH}_{3}(2,2)$ emission (Beltrán et al. 2004; this paper). On the other hand, the following differences have been observed between them: (a) Mid-infrared (3.6, 4.5, 5.8, 8.0, 11.9 and $18.7 \mu \mathrm{m})$ emission has been detected towards G24 A2 using the Spitzer-GLIMPSE survey and VLT-VISIR (see Sect. 3.1); (b) the maximum rotation velocity difference observed in $\mathrm{A} 2\left(1.5 \mathrm{~km} \mathrm{~s}^{-1}\right)$ is less pronounced than that of $\mathrm{A} 1\left(3 \mathrm{~km} \mathrm{~s}^{-1}\right)$ as seen in the $\mathrm{CH}_{3} \mathrm{CN}$ maps (Beltrán et al. 2004); (c) A2 shows weak unresolved radio continuum emission at $1.3 \mathrm{~cm}$ and $7 \mathrm{~mm}$ (Moscadelli et al. 2007), whereas A1 shows the presence of a shell-like bright hypercompact H II region (Beltrán et al. 2007); and (d) fewer water and methanol masers have been observed towards A2 as compared to A1 (Moscadelli et al. 2007). Based on (a) and (b), we speculate that the toroid associated with A2 is possibly less edge-on than that in A1. This would explain the lower velocity gradient in A2 as well as the detection of mid-infrared emission from A2. For systems with identical rotation velocities, a larger inclination leads to a more marked increase in the line-of-sight rotation velocity in comparison to a lower inclination counterpart. We now show that the effect of different masses of A1 and A2 is unlikely to account for the differences in their rotation velocities. Assuming that both A1 and A2 are in rotational equilibrium and that $\mathrm{A} 1$ is close to edge-on given the similar radii and the different masses (130 and $80 M_{\odot}$ respectively for A1 and A2; see Table 1 of Beltrán et al. 2004), one would expect the ratio of the rotation velocities of A2 and A1 to scale as the corresponding ratio of $\sqrt{M}: \frac{v_{\text {rot }}(\mathrm{A} 2)}{v_{\text {rot }}(\mathrm{A} 1)} \simeq \sqrt{\frac{M(\mathrm{~A} 2)}{M(\mathrm{~A} 1)}}$. However, in our case, we find that $\sqrt{\frac{M(\mathrm{~A} 2)}{M(\mathrm{~A} 1)}} \simeq 0.8$ whereas $\frac{v_{\text {rot }}(\mathrm{A} 2)}{v_{\text {rot }}(\mathrm{A} 1)} \simeq 0.5$. This discrepancy can be explained if the toroid in A2 is more inclined than that in $\mathrm{A} 1$, as in this case the observed $v_{\text {rot }}(\mathrm{A} 2)$ would underestimate the true rotation velocity. This is also consistent with the detection of $\mathrm{A} 2$ in the mid-infrared emission because in a less edge-on system, the escaping photons along the outflow cavity are more likely to reach the observer. One can hence speculate that the toroid associated with A2 is more inclined (less edgeon) than that of A1. Most of the differences can also possibly be interpreted in terms of evolutionary stage and/or mass of the central exciting object(s). In other words, if A2 is younger than A1, this would explain the weak radio continuum emission. An alternative is that A2 harbours several lower mass stars (such that the total luminosity of these stars matches the luminosity of A2) than that associated with A1. At present it is impossible to discriminate between the two possibilities on the basis of the available data. Sub-arcsec resolution in the far-infrared will be needed to assess or rule out the presence of multiple deeply embedded stars in regions like this where Av may be as large as 1000 mag.

\section{Summary}

We have probed the nature of the environment of the subcore G24 A2 using infrared, millimetre and radio interferometric measurements. The mid-infrared images from VLT-VISIR and Spitzer-GLIMPSE reveal a single compact source, associated with G24 A2. Weak unresolved radio continuum emission has been detected at $1.3 \mathrm{~cm}$ and $7 \mathrm{~mm}(\sim 0.25)$ which is resolved out at higher angular resolutions (60 mas). This allows 
us to set a limit to the diameter of the radio emitting region of 1000 AU $<D<2000$ AU. Furthermore, the radio spectral index of $\sim 1$ at $\mathrm{cm}$ wavelengths indicates the likelyhood of the radio continuum emission being from a jet or an $\mathrm{H}$ II region with a density gradient. The $\mathrm{NH}_{3}(2,2)$ inversion transition emission from this region peaks slightly north-west of the radio emission. From the $\mathrm{NH}_{3}(2,2)$ spectrum, we observe that while the "main" component looks self-absorbed, this is not the case for the "satellite" component. The velocity distribution (first-moment) maps of the main and satellite component show a clear velocity gradient across the core consistent with the results of Beltrán et al. (2004). Using a simple model of an edge-on disk, we interpret the findings of both $\mathrm{NH}_{3}(2,2)$ (this paper) and $\mathrm{CH}_{3} \mathrm{CN}(12-11)$ $K=3$ (from Beltrán et al. 2004) as the combined effect of expansion and rotation. The observed column densities of $\mathrm{NH}_{3}$ and $\mathrm{CH}_{3} \mathrm{CN}$ obtained from the model are in reasonably good agreement with those obtained from measurements. We have explored scenarios where the expansion is due to outflow(s) from $\mathrm{A} 1$ and/or A2 and we speculate that A2 is the likely origin of the outflow. Finally, we have compared the properties of the neighbours A1 and A2 namely rotation, mass of cores, excitation temperature, infrared and radio emission. Based on these, we conclude that the toroidal structure associated with G24 A2 is possibly more inclined (less edge-on) than that in G24 A1.

Acknowledgements. We thank T. Robitaille for providing us with the midinfrared fluxes at 12 and $18 \mu \mathrm{m}$ (computed from RWIW models) for fitting our data with their models.

\section{Appendix A: Details of the model of an expanding and rotating toroid}

Consider a disk of inner radius $R_{\mathrm{i}}$ and outer radius $R_{\mathrm{O}}$. Figure A.1 shows a schematic of this disk where the $Z$-axis is parallel to the line-of-sight. We assume constant volume density $(n)$ and a temperature dependance on the radius as

$T=T_{\mathrm{o}}\left(\frac{r}{R_{\mathrm{o}}}\right)^{q}$

where $T_{\mathrm{o}}$ is the temperature at the outer radius and $q$ represents the temperature power law index. In our model the disk is undergoing radial expansion and solid-body rotation, which imply the following expressions for the transverse $\left(v_{\theta}\right)$ and radial $\left(v_{\mathrm{r}}\right)$ components of the velocity at an arbitrary point:

$$
\begin{aligned}
& v_{\theta}=v_{\theta \mathrm{o}}\left(\frac{r}{R_{\mathrm{o}}}\right) \\
& v_{\mathrm{r}}=v_{\mathrm{ro}}\left(\frac{r}{R_{\mathrm{o}}}\right) .
\end{aligned}
$$

Here $v_{\theta \mathrm{o}}$ and $v_{\text {ro }}$ represent respectively the rotation and expansion velocities at the outer edge of the disk $R_{0}$. The component of the velocity along $Z$, the line-of-sight, is

$v_{z}=v_{\mathrm{r}} \cos \theta-v_{\theta} \sin \theta$

with $\theta$ defined as in Fig. A.1. Using Eqs. (A.2) and (A.3), this can be rewritten as

$v_{z}=v_{\mathrm{ro}}\left(\frac{z}{R_{\mathrm{o}}}\right)-v_{\theta \mathrm{o}}\left(\frac{x}{R_{\mathrm{o}}}\right)$.

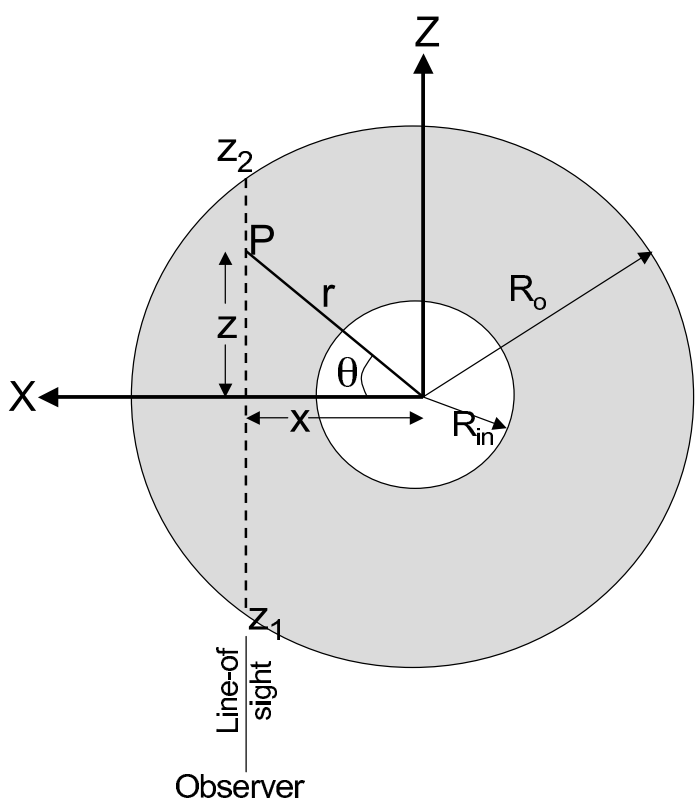

Fig. A.1. Schematic of the model of an expanding and rotating toroid. The $X-Z$ plane represents the disk face-on and contains the line-of-sight to the observer.

The solution of the radiative transfer equation along an arbitrary line of sight gives the brightness temperature at a given velocity, $v$, and position, $x$, along the disk-plane:

$$
\begin{aligned}
T_{B}= & \int_{z_{1}}^{z_{2}} \mathrm{~d} z \kappa J_{v}(T) \mathrm{e}^{-\int_{z_{1}}^{z} \kappa \mathrm{d} z^{\prime}} \quad|x| \geq R_{\mathrm{i}} \\
= & \int_{z_{1}}^{-z_{\mathrm{i}}} \mathrm{d} z \kappa J_{v}(T) \mathrm{e}^{-\int_{z_{1}}^{z} \kappa \mathrm{d} z^{\prime}} \\
& +\left[\int_{z_{\mathrm{i}}}^{z_{2}} \mathrm{~d} z \kappa J_{v}(T) \mathrm{e}^{-\int_{z_{\mathrm{i}}}^{z} \kappa \mathrm{d} z^{\prime}}\right] \mathrm{e}^{-\int_{z_{1}}^{-z_{\mathrm{i}}} \kappa \mathrm{d} z^{\prime}} \quad|x|<R_{\mathrm{i}}
\end{aligned}
$$

where $z_{\mathrm{i}}=\sqrt{R_{\mathrm{i}}^{2}-x^{2}}$ and $J_{v}$ is defined as

$J_{v}=\frac{h v}{k} \frac{1}{\left(\mathrm{e}^{\frac{h v}{k T}}-1\right)}$.

The absorption coefficient is

$\kappa=\frac{c^{3} A_{\mathrm{ul}}}{8 \pi v^{3}} n \frac{g_{\mathrm{u}} \mathrm{e}^{-\frac{h \nu}{k T}}}{Q(T)}\left(\mathrm{e}^{\frac{h \nu}{k T}}-1\right) \phi\left(v-v_{z}(x, z)\right)$.

Here, $h$ is the Planck function, $k$ is the Boltzmann constant, $c$ is represents the velocity of light, $A_{\mathrm{ul}}$ is the Einstein coefficient of the molecular transition of frequency $v, E_{\mathrm{u}}$ is the upper energy level of the transition and $Q(T)$ is the partition function at temperature $T$. We adopt $Q(T)=0.0277 T^{3 / 4}(\mathrm{~K})$ for $\mathrm{NH}_{3}(2,2)$; $Q(T)=0.9741 T^{3 / 4}(\mathrm{~K})$ for $\mathrm{CH}_{3} \mathrm{CN}(12-11)$ transitions and $g_{\mathrm{u}}=2 J_{\mathrm{u}}+1$ represents the statistical weight of the upper level, where $J_{\mathrm{u}}$ represents the rotational quantum number of the level. The partition function has been calculated under the approximation that the rotational constants are small compared to $k T / h$ and with the assumption that all levels are populated according to local thermodynamic equilibrium (Townes \& Schawlow 1975). We considered a Gaussian line profile of the form

$\phi(v)=\frac{2}{\Delta v} \sqrt{\frac{\ln 2}{\pi}} \mathrm{e}^{-4 \ln 2\left(\frac{v}{\Delta v}\right)^{2}}$ 
where $\Delta v$ represents the intrinsic $F W H M$ of the line broadened by thermal effects and microturbulence. The integral in Eq. (A.5) is then computed numerically to produce position-velocity plots along the plane of the disk. For the sake of comparison with the data, plots are then convolved with a 2D Gaussian whose FWHM in the velocity and spatial directions is respectively equal to the channel width and synthesized beam of the VLA.

\section{References}

Anglada, G. 1996, in Radio Emission from the Stars and the Sun, ed. A. R. Taylor, \& J. M. Paredes, ASP Conf. Ser., 93, 3

Beltrán, M. T., Cesaroni, R., Neri, R., et al. 2004, ApJ, 601, L187

Beltrán, M. T., Cesaroni, R., Neri, R., et al. 2005, A\&A, 435, 901

Beltrán, M. T., Cesaroni, R., Codella, C., et al. 2006, Nature, 443, 427

Beltrán, M. T., Cesaroni, R., Moscadelli, L., \& Codella, C. 2007, A\&A, 471, L13

Beuther, H., Churchwell, E. B., McKee, C. F., \& Tan, J. C. 2007, in Protostars and Planets V, ed. B. Reipurth, D. Jewitt, \& K. Keil, 165

Bonnell, I. A., Larson, R. B., \& Zinnecker, H. 2007, in Protostars and Planets V, ed. B. Reipurth, D. Jewitt, \& K. Keil, 149
Carey, S. J., Noriega-Crespo, A., Price, S. D., et al. 2005, BAAS, 1252

Cesaroni, R., Codella, C., Furuya, R. S., \& Testi, L. 2003, A\&A, 401, 227

Cesaroni, R., Galli, D., Lodato, G., Walmsley, C. M., \& Zhang, Q. 2007, in Protostars and Planets V, ed. B. Reipurth, D. Jewitt, \& K. Keil, 197

Codella, C., Testi, L., \& Cesaroni, R. 1997, A\&A, 325, 282

De Buizer, J. M., Radomski, J. T., Telesco, C. M., \& Piña, R. K. 2005, ApJS, 156,179

Fazio, G. G., Hora, J. L., Allen, L. E., et al. 2004, ApJS, 154, 10

Furuya, R. S., Cesaroni, R., Codella, C., et al. 2002, A\&A, 390, L1

Hoare, M. G., Kurtz, S. E., Lizano, S., Keto, E., \& Hofner, P. 2007, in Protostars and Planets V, ed. B. Reipurth, D. Jewitt, \& K. Keil, 181

Indebetouw, R., Mathis, J. S., Babler, B. L., et al. 2005, ApJ, 619, 931

Lagage, P. O., Pel, J. W., Authier, M., et al. 2004, The Messenger, 117, 12

Moscadelli, L., Goddi, C., Cesaroni, R., Beltrán, M. T., \& Furuya, R. S. 2007, A\&A, 472, 867

Palla, F., \& Stahler, S. W. 1993, ApJ, 418, 414

Puget, J. L., \& Leger, A. 1989, ARA\&A, 27, 161

Rieke, G. H., \& Lebofsky, M. J. 1985, ApJ, 288, 618

Robitaille, T. P., Whitney, B. A., Indebetouw, R., \& Wood, K. 2007, ApJS, 169, 328 (RWIW)

Werner, M. W., Roellig, T. L., Low, F. J., et al. 2004, ApJS, 154, 1

Whittet, D. C. B. 1992, J. British Astron. Ass., 102, 230 